Geltung der NS-Repressionsnormen in Frage gestellt. Zu einem Verfahren gegen die jusitiziellen NS-Täter kam es nicht mehr. Immerhin hatte Robert Kempner erreicht, daß die Berliner Staatsanwaltschaft in Vorbereitung des Verfahrens die politischen Strafrechtsnormen des Maßnahmenstaates und ihre Anwendung entlegitimierte.

Als wir 1968 die Kritische Justiz gründeten, war es Robert Kempner, der unser Projekt sogleich unterstützte. Mehrere Beiträge, zur Rolle Hitlers Justizminister Franz Gürtner, zur Exkulpation des Euthanasiearztes Dr. Borm, zur Behandlung des Anstaltsmords im Nürnberger Prozeß, hat er bei uns veröffentlicht. Im ersten Heft der KJ publizierte er, gleichsam programmatisch, eine Rezension der Zeitschrift des Republikanischen Richterbundes »Die Justiz «, für die er in der Weimarer Republik einst selber geschrieben hatte. Er zog eine Linie von der Weimarer zu bundesdeutschen Justiz: »... (S)chon damals (stand) die Schwere der Strafen bei Eigentumsdelikten im krassen Gegensatz zu der Milde gegenüber Massenmördern... Heute können wir auf die milden Strafen für Ingenieure der NS-Todesmaschine hinweisen, die, wenn überhaupt, mit wenigen Jahren Zuchthaus sdavonkamen « (KJ H. I/1968, S. 90).

Robert Kempner negierte die allgemeine Tendenz machtstaatlicher Rechtsentwicklung in Deutschland nach 1933 und z.T. auch nach 1945. Er verkörperte eine Kontinuität rechtsstaatlichen Denkens, das der Allmacht des Staatsapparats entgegenwirkte. Für die Justiz, dem großen Handlungsfeld Robert Kempners, bleibt dies ein wichtiges Postulat.

\title{
Heidemarie Renk Erste Erfahrungen mit dem neuen Betreuungsgesetz
}

Am I. 1.1992 trat das neue Betreuungsgesetz (BtG) in Kraft. Die Vorbereitungsarbeiten dauerten fast zwanzig Jahre. Schon 1975 wurden die wesentlichen Mängel des zuvor geltenden Vormundschafts- und Pflegschaftsrechts in einem Bericht zur Lage der Psychiatrie aufgelistet (BT-Drs. 7/4200, S. $307 \mathrm{ff}$.). 300 Paragraphen in über 50 Gesetzen wurden im Zuge dieser Reform geändert. Dem Aufwand entsprechend war auch die positive Aufnahme in der Fachwelt und der Öffentlichkeit. Gefeiert wurde das BtG als das » wichtigste rechtspolitische Vorhaben der letzten Jahre« (Bundesministerin der Justiz Leutheusser-Schnarrenberger). "Das Gesetz eilt in mancherlei Hinsicht seiner Zeit voraus; es konkretisiert aus unserem Grundgesetz gewachsene Wertmaßstäbe, die noch nicht fest genug in unserem Bewußtsein verankert sind. Es wird unserer aller gemeinsamer Anstrengungen bedürfen, den Geist des Gesetzes zur sozialen Wirklichkeit werden zu lassen. «

Das neue Gesetz legt seinen Schwerpunkt der Hilfen für Menschen, die aufgrund einer psychischen Krankheit oder einer körperlichen, geistigen oder seelischen Behinderung ihre Angelegenheiten nicht mehr ganz oder teilweise besorgen können ( $\$ 1896$ I BGB), zum einen auf die persönliche Betreuung, zum anderen auf die geringstmögliche Einschränkung der Handlungsfähigkeit. Dadurch sollten insbesondere dem Schutz der Menschenwürde und der Selbstbestimmung des Individuums Rechnung getragen werden. In den Hintergrund traten die früheren Motive,

\footnotetext{
I Aus dem Vorwort eines vom Bundesministerium der Justiz 1992 herausgegebenen Leitfadens »Das Be-
} treuungsgesetz in der Praxis", Verlag Bundesanzeiger. 
nämlich der Erhalt des Vermögens für die Familie sowie der Schutz des Rechtsverkehrs durch eine klare Ausgrenzung der konstitutiv festgestellten Geschäftsunfähigen. Wurden die neu gesetzten Schwerpunkte anfangs begrüßt, dominiert inzwischen die Klage über die bescheidene Umsetzung. Es fehlen die notwendigen ehrenamtlichen Betreuer, die nach der Vorstellung des Gesetzgebers eine wichtige Rolle spielen sollen. Es fehlt weiter an den erforderlichen finanziellen Mitteln, etwa um Betreuungsbehörden einzurichten bzw. diese auszubauen oder ehrenamtliche Betreuer zu finden. Nach zweijähriger praktischer Erfahrung häuft sich aber auch die Kritik an der Konzeption des Gesetzes selbst. Um dessen materiellen Grundlagen genügen zu können, hat der Gesetzgeber eine Fülle von Verfahrensvorschriften eingeführt. "Der nachfolgende Aufsatz gibt Erfahrungen und Eindrücke aus der ersten Anwendungsphase unmittelbar nach Inkrafttreten des Betreuungsrechts wieder. Er will dazu anregen, auch bei dieser gerade erst neu geschaffenen Verfahrensordnung über Verfahrensvereinfachungen nachzudenken, die nach Ansicht der Autorin möglich wären, ohne dem Betreuungsrecht in der Sache zu schaden.»

\section{Erwartungen an die Reform}

Die Reform des Rechts der unter Vormundschaft und Pflegschaft Stehenden war Projekt im wesentlichen derer, die, sei es professionell oder unentlohnt, mit davon Betroffenen verbunden waren bzw. sind. Programmatisch stand im Mittelpunkt, Freiheitsrechte und Selbstbestimmung zu betonen und fürsorglichen $Z$ wang zurückzudrängen. In diesem Sinne kann es als Intention des Betreuungsrechts gesehen werden, die rechtliche Form eines Problembewußtseins dafür zu schaffen, daß die vermeintlichen Wohltaten der Helfer gelegentlich zur "aufgedrängten Bereicherung « gerieten, die die Betroffenen selbśt nicht wollten und bei denen die Unterstellung, sie lägen in deren objektivem Interesse, die Betroffenen gar zu sehr vom Lebensentwurf der Helfer abhängig machten. Von dieser Zielsetzung her ${ }^{2}$ sind die wesentlichsten Charakteristika des Gesetzes zu sehen, auf deren Kodifizierung von Anfang an der Schwerpunkt lag: Nur das im Entscheidungsmoment Nötigste sollte veranlaßt werden können, abgesichert und begleitet durch ärztliche Gutachten und stets und regelmäßig erneuter Prüfung unterzogen. Maßstab der jeweiligen Entscheidung sollte neben immer schon fragwürdiger Fremdbeurteilung eines »objektiven Interesses« des Betroffenen dessen Wunsch und Wille selbst sein. Soweit vereinzelt gehofft wurde, das Verfahren, mit dem rechtliche Angelegenheiten des Personenkreises des heutigen $₫$ I $896 \mathrm{BGB}$ geregelt würden, könne vereinfacht und entbürokratisiert werden, hat sich dieses Interesse im Ergebnis nicht durchsetzen können.

\section{Der Wille des Gesetzgebers}

Neben den motivationalen Hintergründen der oben angesprochenen Reformdebatte, die auf seiten des Gesetzgebers im wesentlichen anerkannt und berücksichtigt wurden, findet sich in der Begründung des Regierungsentwurfs zum Betreuungsgesetz auch der in einer Reformgesetzgebung allgemein verbreitete Topos, wonach der ohnehin durch die Praxis der Rechtsprechung gewählte Weg zu kodifizieren sei; insoweit wird unter Rekurs auf einschlägige Zahlen das Phänomen angesprochen, daß es eine ständig sinkende und gegen Null tendierende Zahl von Entmündigten gab, während man bereits im Rahmen der Pflegschaft mit milderen Mitteln den

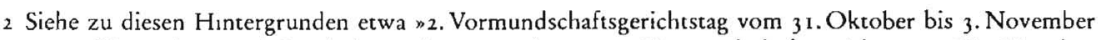
1990; Materıalıen und Ergebnisse", herausgegeben vom Vormundschaftsgerichtstag e.V., Munchen 1991. 
Zustand zu erreichen versuchte, der vordem im Wege der Entmündigung hergestellt wurde ${ }^{3}$. Keineswegs unberücksichtigt blieb der Kostenfaktor: Vermieden werden sollten gar zu hohe Kosten zu Lasten der Staatskasse, weshalb bei bestimmten Einzelmaterien von grundlegenden Änderungen abgesehen wurde ${ }^{4}$. Der Kern der Neuerung aber liegt im ideellen Bereich, dem, der Rechte und Pflichten konstituiert, ohne unmittelbar Geldströme abzuschöpfen: Rechtsstaatsgarantien sind insoweit ausgebaut, als es um die Statuierung von Informationspflichten, von einzuschaltenden Personen oder Institutionen, von Rechtsmitteln geht. Verfahrensgarantien als der klassische Rechtsschutz der aufgeklärt-demokratischen Gesellschaft - Verfahrensgarantie als das (rechts-)staatliche Versprechen, den Inhalt einer Entscheidung im Rahmen einer Prozedur zu entwickeln, die maximale Kritik und damit maximale Richtigkeit zuläßt - sind als auf den ersten Blick wohlfeile Angebote des Rechts- und Sozialstaats an je passender Stelle gesetzt und verankert.

\section{Erste praktische Erfabrungen}

Jede größere Gebietskörperschaft dürfte über Richter, Sozialarbeiter, Psychiater, Betroffenenverbände und ehrenamtliche Helfer verfügen, die für sich in Anspruch nehmen, das neue Betreuungsrecht schon praktiziert zu haben, ehe es als Gesetz in Kraft trat. Das alte Recht stand dem nicht grundsätzlich entgegen. Dennoch ist die Euphorie, mit der allseits dem Betreuungsrecht entgegengesehen wurde, in dessen Vollzug nicht (mehr) angebracht. Die »grundsätzliche" Verbesserung, die die Reform versprochen hat, ist nicht eingetreten. Gerade aus der Sicht derer, die gewinnen sollten, der Betroffenen, haben die gesetzgeberischen Eingriffe insbesondere wegen des hohen Maßes an Inflexibilität des zur Verfügung stehenden Instrumentariums im einzelnen vielleicht Fortschritte, per saldo aber kaum einen tatsächlichen Gewinn gebracht. Allein die unausweichliche Komplexität des Verfahrens hat aber beeinträchtigenden Charakter, so daß im quantifizierenden Ergebnis unter dem Strich die Nachteile überwiegen dürften.

Dies soll in der Folge an verschiedenen Einzelaspekten des "gewöhnlichen« Betreuungsverfahrens demonstriert werden:

Die detailfreudigen Normen des Betreuungsrechts bieten ein fein abgestimmtes Repertoire an Verfahrensregeln, die den Weg der Verwertung materiell-rechtlich relevanter Befunde wissen. Minutiös wird jeder Schritt des Betreuungsverfahrens geregelt:

Eine Betreuung wird angeregt. Die Richterin führt ein Erstgespräch, in dessen Verlauf sie sich sowohl der Wünsche des Betroffenen wie auch des Betreuungsbedarfs vergewissert. Zu genauerer Aufklärung der Sache wird die Betreuungsbehördes eingeschaltet. Diese recherchiert - etwa durch Rückfrage beim bisher mit der Angelegenheit befaßten Sozialdienst - die zugrundeliegenden Sozialdaten und erstellt einen Sozialbericht. Parallel dazu wird ein Gutachten über die Voraussetzungen der Betreuung nach $\$ 1896$ BGB eingeholt. Dieses Gutachten erstattet der psychiatrisch qualifizierte Arzt unter Angabe nicht nur der Befunde, sondern auch der Explorationstechnik und der sonstigen angewandten diagnostischen Mittel. Es nimmt sorgfältig nicht nur zu den Krankheitsvoraussetzungen, sondern auch zur Möglich-

3 Siehe Begründung des Regierungsentwurfs vom 11.5. 1989, BT-Drucks. 11/4528, S. 42 ff.

4 Ebenda, S. 94; verworfen wurde der Gedanke der allgemeinen Kostenfresheit des Verfahrens zugunsten diverser Einzelregelungen.

s Soweit vorhanden: Wie der Frankfurter Lokalpresse vor einiger Zeit zu entnehmen war, verzichtet die Stadt nun kunftig offensiv und programmatisch auf Beseitigung des bisher vielfach beklagten Fehlens einer einsatzfăhigen Behörde. 
keit des Einsatzes anderer Mittel als der Betreuung Stellung, ebenso dazu, wie lange voraussichtlich der Betreuungsbedarf vorliegt und ob eine Rehabilitationsmöglichkeit besteht. Soweit mit dem Betroffenen der Ablauf des Betreuungsverfahrens nicht erörtert wurde oder werden konnte, erhält dieser inzwischen schriftliche Mitteilungen seitens des Gerichts, welche Ereignisse (etwa: Besuch durch Mitarbeiter der Betreuungsbehörde und des Gesundheitsamts) auf ihn zukommen. Er wird umfassend über seine Rechte informiert (etwa, daß er eine Anhörung in seiner gewohnten Umgebung nicht zu akzeptieren braucht oder daß er eine Person seines Vertrauens hinzuziehen oder Prozeßkostenhilfe beantragen kann). Hat sich der Betroffene beim Erstgespräch oder nach Aktenlage als ein Mensch erwiesen, bei dem eine Anhörung nicht möglich ist, so wird ihm alsbald ein Verfahrenspfleger zur Wahrnehmung seiner Rechte bestellt. Ebenso, falls sich abzeichnet, daß die Betreuung umfassend sein muß $\beta^{6}$. Alsdann liegen Gutachten und Sozialbericht vor; die Richterin kann nunmehr mit dem Betroffenen das Schlußgespräch führen. Für maximal fünf Jahre wird nun die Betreuung errichtet, dann wird sie erneut überprüft, wobei das vorstehend geschilderte Verfahren in jedem einzelnen Punkt erneut durchlaufen wird. Eine frühere Wiederholung dieser Verfahrensschritte findet dann statt, wenn - wie häufig und vom Betreuungsrecht gerade verlangt - nach Errichtung der Betreuung weiterer Betreuungsbedarf entsteht, der zuvor zwar bereits vorauszusehen, aber noch nicht aktuell gegeben war.

Anwendungsbeispiele:

Ein 83 Jahre alter Rentner lebt allein und wird regelmäßig von zwei Töchtern besucht und versorgt. Mittags erhält er Essen auf Rädern. Die Töchter kümmern sich um die Wohnung, um Kleidung, um Wäsche. Da sie berufstätig sind, können sie allenfalls einmal am Tag vorbeikommen. Seit einiger Zeit berichten die Nachbarn darüber, daß der Betroffene immer wieder einmal nachts durch das Haus irrt, bei ihnen klingelt, unzureichend bekleidet ist und verwirrt wirkt. Eines Tages kommt eine der Töchter gerade noch rechtzeitig, um einen Brand zu verhüten, nachdem durch unkontrollierten Umgang mit der Gasflamme bereits Feuer ausgebrochen war. Der Betroffene wollte, allein und hilflos, mit der Gasflamme heizen. Binnen weniger Tage steht ein Heimplatz zur Verfügung. Der Betroffene könnte sofort umziehen, wenn ein Heimvertrag geschlossen und die Kostenfrage geregelt würde. Der Hausarzt bescheinigt »senile Demenz und Desorientiertheit in allen Qualitäten «. Bei der Anhörung ist ein freundlicher alter Herr zu finden, mit dem ein sinnvolles Gespräch nicht mehr möglich ist und der nicht anzugeben weiß, wo er sich befindet. - Ist hier das Gutachten eines psychiatrisch erfahrenen Arztes zwingend?

- Warum ist die kurzgutachterliche Stellungnahme des Hausarztes unzureichend? Ein 28 Jahre alter geistig Behinderter bedarf eines minimalen operativen Eingriffs. Bisher wurde, weil nur soweit Bedarf bestand, lediglich die Vermögenssorge und das Aufenthaltsbestimmungsrecht als Pflegschaftsrechtskreis geführt. Betreuerin ist die Mutter des Betroffenen. Der »unmittelbare Eindruck « zeigt auf den ersten und den zweiten Blick einen nicht kommunikationsfähigen und sichtlich nicht zur eigenen Entscheidung befähigten Menschen. Ein Gutachten wird (gleichwohl) eingeholt. Die Betreuung wird um den Aufgabenkreis Gesundheitssorge erweitert.

- Muß ich hier einen Verfahrenspfleger haben, der die Rechte des Betroffenen vor dem Betreuer und dem Gericht schützt?

6 Es bleibt dabei der richterlichen Gewissensentscheıdung uberlassen, ob Verfahrenspfleger und Betreuer in einer Person zusammenfallen; trotz zunachst gegenteiliger Bestrebungen ist diese Moglichkeit durch das $\mathrm{BtG}$ eingeraumt worden. 
Ein Altenpflegeheim teilt mit, es müsse bei Frau X ein Sicherungsgurt angelegt werden, wenn sie im Rollstuhl säße. Zwar könne sie ohne sog. unterbringungsähnliche Maßnahmen im Bett liegen, doch solle sie am Tagesgeschehen des Heims teilnehmen, um sie zu aktivieren, was nur durch die beschriebene Sicherheitsmaßnahme möglich sei. Man wolle auch regelmäßig und nicht nur sporadisch in dieser Weise verfahren.

- Soll Frau X bis zur mehrwöchigen Abwicklung der Betreuungsprozedur »frei beweglich « im Bett liegen bleiben?

Kurz nach Errichtung einer Betreuung - das eingeholte ärztliche Gutachten, aus dem hervorgeht, daß es sich um einen nicht mehr rehabilitationsfähigen Zustand handelt, ist ein Vierteljahr alt - kristallisiert sich weiterer Betreuungsbedarf heraus - etwa die Notwendigkeit zu einer Postempfangsbefugnis des Betreuers, weil finanzielle Dinge geregelt werden müssen und in Zusammenhang damit mit Posteingang zu rechnen ist.

- Welche neuen Erkenntnisse sollten einem weiteren ärztlichen Gutachten noch zu entnehmen sein?

Es ist nicht alleine das Problem, daß das Betreuungsrecht entgegen der ursprünglich gehegten Hoffnung doch Geld kostet (weil es Richter für die aufwendigen Verfahren braucht, weil der Gutachter kostet, weil die Stellen der Betreuungsbehörde bezahlt werden müssen), schwerer wiegt, daß es unendlich viel Zeit in Anspruch nimmt, ohne daß diesem Zeitaufwand ein entspechender Erkenntnisgewinn gegenüberstünde. Die oben skizzierten Fälle sind Alltag der Vormundschaftsrichter. Schon nach kurzer, Erfahrungen generierender Zeit läßt sich sagen: In der Mehrzahl aller Fälle bedarf es weder zweier Gespräche noch des Gutachtens eines psychiatrisch erfahrenen Arztes, um den Betreuungsbedarf zu erkennen, ebenso, daß dieser durch anderweitige Hilfsmittel nicht auszugleichen ist und Rehabilitationsmöglichkeiten ausscheiden. Auch werden die Rechte des Betroffenen keineswegs in demselben Umfang besser gewahrt, in dem die Zahl der dafür Verantwortlichen erhöht wird. Statt dessen sollte oftmals rasch gehandelt werden. Indes: Das Betreuungsrecht steckt voller zwingender Regelungen, bietet aber die Möglichkeit zu raschem $\mathrm{Han}$ deln nur äußerst eingegrenzt. Verfahrensregeln werden nicht differenziert nach dem je verschiedenen Betreuungsfall, Ermessensspielräume sind kaum vorhanden. Gerade der Regelfall, die »unproblematische« Betreuung, wird überkomplex mit zwingenden Vorschriften überzogen, die bei kritischen Fällen wichtig, bei klaren Fällen zur Belastung für alle Beteiligten werden. Rasches Handeln, entschließt man sich denn dazu, bedeutet Handeln im »Notfall«-Bereich: Mit dem nach wie vor anwendbaren $\mathbb{I}_{1846} \mathrm{BGB}$ habe ich das - mit Recht für bedenklich gehaltene ${ }^{7}$ - formale Mittel, ohne Betreuer und einstweilig nahezu alles anzuordnen, was prima facie als sinnvoll erscheint. Damit schafft das Betreuungsrecht eine paradoxe Situation: Je sorgfältiger des Betroffenen Rechte gewahrt werden, indem das Betreuungsverfahren in allen Einzelschritten durchlaufen wird, desto eher verlagern sich sachgerechte Lösungen in die ferne Zukunft. Wenn der Gesetzgeber - erkennend, daß zumindest für Notfälle Sorge getragen werden muß - diesem ausgefeilten Instrumentarium keine Abstufungen implementiert, innerhalb derer von einzelnen Maßnahmen nach richterlichem Ermessen Gebrauch gemacht werden kann, sondern lediglich durch Einbau einer keinerlei Verfahrensgarantien mehr enthaltenen Generalklausel Ausweichmöglichkeiten schafft, bringt er geradezu systematisch die Rechtsanwender in Konflikt. Dieser Konflikt ist auch keineswegs ein lediglich subjektiver; die vom Verfahren Berührten (etwa Sozialarbeiter, denen an einer raschen Lösung gelegen ist,

7 So das am - zu Recht - mißbilligenden Ausrufungszeichen erkennbare Votum des Frankfurter Vormundschaftsrichters Rink in einem gerichtsinternen Leitfaden zur Behandlung vormundschaftsgerıchtlicher Eilfalle. 
weil sie einen Heimplatz entweder jetzt gleich oder gar nicht haben) drängen massiv auf umgehende Entscheidung: Regelmäßig wird das Gericht dazu aufgefordert, man möge doch in diesem eindeutigen Fall eine einstweilige Anordnung erlassen, da die Sachlage keiner Ermittlung mehr bedürfe und ohnehin auf der Hand liege.

Aus alledem ergibt sich die Schlußfolgerung: Das »neue Recht« ist ein umständliches, aufwendiges und bürokratisch überkomplexes Verfahren. Es scheint auszugehen vom Regelfall des Irrtums: Jedwede Entscheidung wird flankiert (von Gutachten), reflektiert (im Hinblick auf die Rechtsmittelmöglichkeiten der verschiedenen Beteiligten), vervorläufigt (aufgrund des Erfordernisses regelmäßiger Überprüfung). Flexibilität der zu treffenden (Verfahrens-)Maßnahmen wird zurückgedrängt, weil sie unter allerlei Verdacht steht, etwa dem, sich keiner Mühe unterziehen zu wollen, oder dem, sich vorschnell seiner Sache sicher zu sein. Es wird schlichtweg geleugnet, daß es in diesem Bereich zahlreiche Situationen gibt, die so weit außer Streit stehen, daß der - auch die Betroffenen eingreifend tangierende - Aufwand des mißtrauischen Betreuungsrechts überkomplex gerät. Ein Beispiel: Das Vormundschaftsgericht braucht - nach einschlägiger Kommentierung ${ }^{8}$ - das Gutachten eines psychiatrisch erfahrenen Arztes selbst dann, wenn der internistisch, chirurgisch oder geriatrisch qualifizierte behandelnde Arzt einen Befund bietet, den auch die/der Richter/in selbst zu erfassen imstande ist (z. B. dauerhafte Bewußtlosigkeit oder erkennbare und lebenslange schwere geistige Behinderung). Weiter geleugnet wird der Umstand, daß solche klar erkennbaren Situationen ${ }^{9}$ den Regelfall bieten. Die Imagination des handlungsfähigen Subjekts - das in der Praxis jedenfalls (und wohl nicht nur) im Betreuungsrecht verschwindend selten ist - führt so weit, daß das Betreuungsrecht bei seiner Konzeption aus den Augen verloren zu haben scheint, daß die von ihm Gemeinten wohl nur selten diejenigen sein werden, die dessen Verästelungen - auf dem Weg der Beschwerde oder auf einem anderen Weg - nachzuvollziehen in der Lage sein werden. Schon immer galt, daß die Mündigkeit des Bürgers dessen Informiertheit voraussetzt; erst recht gilt dies offenbar im rechtsstaatlich-subjekthaft orientierten Betreuungsrecht, das ganz besonders viel Informiertheit verlangt, um die vielfältigen Verfahrensvorschriften in ihrer inneren Logik erfassen zu können.

Um einem möglichen Mißverständnis vorzubeugen: Es sind nicht die oben beschriebenen Reformintentionen, denen die Kritik gilt. Tatsächlich weisen diese in eine Richtung, die ebenso zeitgemäß wie sozialadäquat ist. Ohne jeden Zweifel war ein weiterer Schritt aus der "Kuratel « fällig und war es an der Zeit zu einer Entrechtlichung. Die vormalige Zugriffsweise, nach der oftmals rasch und oftmals umstandslos ohne ausreichende Ermittlung der Voraussetzungen und des Bedarfs eine Pflegschaft eingerichtet wurde, um deren Fortgang einschließlich des Betroffenen selbst sodann unter im wesentlichen vermögensorientierten Gesichtspunkten streng zu überwachen, entsprach mehr den Lebensverhältnissen zu Zeiten des Allgemeinen Preußischen Landrechts als den heutigen, in denen sich die Erkenntnis verbreitet, daß das Lebensrecht eines Menschen auch darin bestehen kann, sich nicht in bestmöglicher Weise zu versorgen oder zu fördern, und auch darin, in einem Lebenszusammenhang belassen und betreut zu werden von nahestehenden Menschen, die Vermögensverwaltung allenfalls suboptimal beherrschen. Hier die inquisitorischen Kontrolleingriffe der Vormundschaftsgerichte zurückzuschneiden, die mit besten Absichten,

8 Damrau/Zimmermann, Kommentar zum BtG, $\$ 68 \mathrm{~b}$ FGG, Rdnr. 7 .

9 Solche klare Erkennbarkeit der Situation wurd schließlich auch aus den engeholten Gutachten und den dort mitgeteilten Diagnosen ersichtlich: die Standarddıagnose beim alteren Betroffenen lautet "senile Demenz aufgrund hirnorganischen Psychosyndroms und vermag nach kurzester Zeit nicht mehr uberraschen. 
aber ohne Berücksichtigung individueller Lebensgestaltung die allseitige Absicherung in den Vordergrund stellten, kann nur mit Nachdruck begrüßt werden. Indes ist dieser Aspekt der Entrechtlichung - die es im übrigen erst noch gegen viele Widerstände ins Werk zu setzen gilt (der fürsorgliche Zwang liegt dem Helfer oder sonst Verantwortlichen oft deshalb näher, weil er dadurch des Risikos enthoben ist, Fehler durch Nichthandeln zu begehen) - nur ein Aspekt der Neuregelung; neben diesen Aspekt sind umfängliche Verrechtlichungsphänomene getreten, die in anderer Richtung übermäßig belastend wirken und im Grunde nur dann sinnvoll sein könnten, wenn sie von Bedeutung für das Ergebnis des Verfahrens wären. Gerade das aber sind sie nicht. Bei den vielen Elementen des Betreuungsverfahrens handelt es sich in der weit überwiegenden Mehrzahl aller Fälle zwar um notwendig zu absolvierende Stationen, ungeachtet dessen kann das Durchlaufen dieser einzelnen Stationen sehr häufig ersichtlich nicht von Einfluß sein, weil es sich um jene von mir oben skizzierten Situationen handelt, in denen bereits durch Einsatz sehr viel geringerer Mittel hinreichend festgestellt werden kann, welche Entscheidung zu treffen ist. Dies hat zur Folge, daß die Verfahren trotz aller vom Gesetzgeber vorgesehenen Prüfmomente in zahlreichen Fällen keine Wendung nehmen und die Prüfung deshalb im Ergebnis als überflüssig erscheint, zumal dieser Umstand von vorneherein ersichtlich ist. Wenn Verfahrenselemente eingebaut werden, ohne daß empirische Erfahrungen dafür sprechen, daß sie sinnvolle Entscheidungshilfe sein können, dann liegt eine schlichte Fehlkonzeption vor, und gerade dies, so meine Behauptung, ist im Betreuungsrecht der Fall ${ }^{10}$.

Bleibt zuletzt die Frage, für wen das Betreuungsrecht in der vorliegenden Form wohl konzipiert wurde, wenn nicht für die Fälle, in denen es als überkomplex imponiert. Hier wäre meines Erachtens in erster Linie an die sozial Auffälligen und an die psychotisch Erkrankten zu denken.

Für die sozial Auffälligen, die handlungsfähig und zugleich krankheitsbeeinträchtigt sind, aber ungeachtet dessen sich ihre Rechte nicht nehmen lassen wollen, weil sie es als ihre Sache ansehen, ob sie zurechtkommen oder nicht, scheint das Betreuungsrecht in erster Linie konzipiert. Hier ist es auch sinnvoll in allen Verästelungen. Es ist sinnvoll, immer wieder zu prüfen, wo krankheitsbedingte Ausfälle enden und freie Willensbestimmung beginnt. Sinnvoll auch, verschiedene Verfahrensstufen nacheinander zu schalten, um die Situation von allen Seiten zu beleuchten. Indes: Man darf nicht aus den Augen verlieren, daß diese Gruppe der Betroffenen zahlenmäßig sehr klein sein dürfte und es sicher nicht rechtfertigt, für sie sinnvolle Maßnahmen gegenüber allen Betroffenen zum zwingenden Repertoire zu machen.

Daneben gibt es die psychotisch Erkrankten. Auch für diese ist das Betreuungsrecht ein grundsätzlich geeignetes Instrumentarium, wobei hier hinzutritt, daß häufig

ıo Daß daneben auch im Detail nicht ganz zu Ende gedacht wurde, zeigt die mißliche Sıtuation, in die der Vormundschaftsrichter kommen kann, will er allen Vorschriften in wörtlicher Auslegung Rechnung tragen: Das Grundgesetz sieht in Art. 104 vor, bei Freiheitsentzug am auf den Begınn des Freiheitsentzugs folgenden Tag den Betroffenen dem Richter vorzufuhren, der »unverzüglich* eıne Entscheidung zu treffen hat. Das BtG sieht hinsichtlich des Wirksamwerdens eines Unterbringungsbeschlusses mit sofortiger Vollziehbarkeit vor, daß dieser der Geschaftsstelle zum Zwecke der Bekanntmachung ubergeben worden sein muß $(\$ 7$ og Abs. 3 S. 3 FGG). Man fuhre sich die praktuschen Probleme vor Augen, die dadurch entstehen, daß man Anhórungen in diesen Fällen noch bis Mitternacht durchfuhren kann, dem Betroffenen sodann den Beschluß gemäß $\$$ 7og Abs. I FGG bekannt gibt, dessen Wırksamkeit gleichwohl aber - statt mit Verkündung - erst dann eintreten kann, wenn die Geschaftsstelle besetzt und der Beschluß dort angelangt ist. Für einen gerichtlichen Wochenend-Eildienst ist das entstehende Dilemma praktisch unlösbar. (Keine Lösung dürte die Frankfurter Arabeske sein, wonach man den Frıstenkasten des Gerichtsgebaudes kurzerhand zur Geschaftsstelle erklart hat und es dem Eilrichter aufgibt, nach der Anhorung am Unterbringungsort zu Gericht zurückzukehren und Akte nebst Beschluß dort $1 \mathrm{~m}$ Brıefkasten zu deponieren.) 
kurzzeitige Regelungen erforderlich sind. Dies hat ein besonders eigentümliches Phänomen zur Folge: den Richter als Teil des Behandlungskonzepts. Um zu verstehen, was dies bedeutet, mag man sich die Situation einer Unterbringung vorstellen, die vordem (in Hessen) im wesentlichen über das HFEG bewerkstelligt wurde. Nach wie vor werden Leute dabei angetroffen, wie sie kurz davor stehen, andere anzugreifen ("Fremdgefährdung «), sich selbst in krankhaftem Zustand, etwa auf der Flucht vor vermeintlichen Verfolgern, durch aberwitzige Aktionen, etwa den Sprung aus dem Fenster, zu schädigen ("Eigengefährdung "). Daneben finden wir Patienten im ganz offenbaren psychotischen Schub, die dringend der (medikamentösen) Hilfe bedürfen, weil sie ohne diese absehbar tiefer und tiefer in die Psychose gleiten würden, ohne daß ihre Krankheit ihnen erlaubte, über die Behandlung eine Entscheidung zu treffen. Zu prüfen bleibt die Rechtfertigung der "Zwangsbehandlung «, und es zeichnet sich - jedenfalls nach meiner subjektiven Erfahrung - in diesem Bereich ab, daß Richter für die Psychiater Entlastungsfunktion bekommen. Man begrüßt es, daß zumindest auch die Richter, gleich wie die Entscheidung ausgeht, die Verantwortungsträger sind. Womit einhergeht, daß mit dieser Delegation Personen in die Verantwortung geholt werden, die wiederum fachlich dazu nicht qualifiziert sein können. Das ist dem Richteramt zwar nicht fremd (im Prinzip erfährt man dies bei jeder Sachverständigenanhörung), neu aber ist, daß es mit dieser Häufigkeit und Regelmäßigkeit geschieht. Hält man sich an die Maximen des Betreuungsrechts, wonach kurzfristig nur dem derzeitigen Regelungsbedarf entsprochen werden soll und Vorsorgebetreuungen oder -unterbringungen auch für kürzeste Zeiträume zu unterbleiben haben, so wird man bei der kleinsten Veränderung immer wieder in die Sache einbezogen und vor Ort erscheinen. Wenn dies nun, wie vom Betreuungsrecht durchaus vorgesehen, mehrmals geschieht, wird die Rolle der Richter zweideutig: Man wird Teil eines Konzils. Man beurteilt nicht mehr ex post einen Sachverhalt, sondern lebt in ihm und produziert ihn. Entscheidungen werden im Rahmen einer gemeinsamen Beratung aller Beteiligten getroffen. Zwangsläufig fließen zahlreiche Gesichtspunkte in solche Entscheidungsfindungsprozesse ein; man wird nicht nur mit dem jeweiligen Krankheitsbild und dem daraus resultierenden Fürsorgebedürfnis vertraut, sondern auch mit strukturellen Versorgungsmängeln wie etwa dem, daß nur auf den geschlossenen Abteilungen die Ausstattung mit Pflegekräften so ist, daß der nicht Unterbringungsbedürftige, aber pflegerisch anspruchsvolle Betroffene adäquat versorgt werden kann. Da ein solcher Zustand weder vom Gericht noch von der jeweiligen Klinikverwaltung mit Wirkung auf den konkreten Fall beeinflußt werden kann, steht man vor der Alternative, durch striktes Festhalten am, geltenden Recht den Betroffenen minderversorgt zu wissen oder gesellschaftlich hingenommenen Pflegenotstand durch richterlichen Beschluß zu legalisieren und damit zu perpetuieren.

Ob das neue Betreuungsrecht sich in diesem Bereich bewährt und welche Auswirkungen eine solcherart veränderte Aufgabenstellung auf die damit befaßten Richter und Gerichte hat, bleibt abzuwarten. Wichtig scheint mir in diesem Zusammenhang die Überlegung, daß zwar einerseits die gestiegene Sensibilität für Eingriffe in Freiheitsrechte zu begrüßen ist, dies andererseits aber nicht dazu führen darf, solche Eingriffe schon an der Quelle auf ihre Verhältnismäßigkeit zu kontrollieren (durch eine neue Konzeption der Pflege und Versorgung der Betroffenen, die ohne Rechtsverlust Abhilfe zu schaffen in der Lage ist). Auch hier ist einer der Punkte, an denen die Vormundschaftsgerichte unter zum Teil erheblichen Erwartungsdruck gesetzt werden: Es wird oftmals der Wunsch geäußert, man möge - z. B. bei freiheitsbeschränkenden Maßnahmen wie Bettgittern- rasch entscheiden, um die ohnehin exekutierte Praxis zu legitimieren. 
Es drängt sich der Eindruck auf, als seien viele dieser konkreten Alltagssituationen nicht hinreichend bedacht worden und als habe man im Taumel der Freiheitsrechte vergessen, sich die gefundenen Regelungen in ihrer praktischen Anwendung vorzustellen. In diesem - negativen - Sinne ist das Betreuungsgesetz idealistisch.

Zusammenfassend bleibt festzuhalten:

a) Das Betreuungsrecht ist ein im Konzept sinnvolles, in der Umsetzung ohne Not überreguliertes Recht.

b) Ohne Einschränkung zu begrüßen ist die Wegorientierung von der staatlich kontrollierten Vermögensverwaltung für "Mündel « oder »Pflegling « (in diesem Zusammenhang auch die Konzeption der Einheitsentscheidung und die Übertragung der Zuständigkeit der Betreuerauswahl auf den Richter) und von der Betreuung dort, wo ein Bedarf hierfür nicht besteht.

c) Abzulehnen ist die zwingende Anordnung des Gesetzgebers, daß jedes Betreuungsverfahren dieselben umfänglichen Tatsachenermittlungsschritte durchlaufen muß, ohne es dem Richter - wie in anderen Prozeßordnungen - an die Hand zu geben, welcher Aufklärungsbedarf konkret besteht. Der Umstand, daß nach altem Recht tatsächlich zu wenig der Betreuungsbedarf ermittelt und zu schnell die Pflegschaft eingerichtet wurde, sollte nicht dadurch kompensiert werden, daß nun auch die eindeutigen Fälle mit Verfahrensschritten belastet werden, bei denen von vorneherein ersichtlich ist, daß sie auf das Ergebnis des Verfahrens keinen Einfluß haben werden.

d) Abzuwarten bleibt die Erfahrung mit einer »begleitenden « Justiz, die kraft Amtes an der Rehabilitation eines Erkrankten mitzuwirken hat.

\section{Nachtrag}

Bis hierher war von ersten Erfahrungen mit dem neuen Betreuungsrecht die Rede: Mittlerweile dürfte von weiteren Erfahrungen gesprochen werden können. Die Errichtung der Betreuungsvereine geschieht bestenfalls schleppend, den vorhandenen Organisationen fehlen vielfach die personellen und sachlichen Mittel zur Umsetzung ihrer Aufgaben; die Arbeit der Betreuungsbehörde ist vielerorts nicht zufriedenstellend, da infolge des finanziellen Engpasses der schwerpunktmäßig belasteten Kommunen die erforderlichen Finanzmittel nicht bereitgestellt werden können, die zur Durchsetzung des Reformkonzepts benötigt würden. Aber auch die Justiz wird wie sich dies immer deutlicher abzeichnet - kaum in der Lage sein, das Betreuungsrecht so umzusetzen, wie es der Gesetzgeber konzipiert hat. In Zusammenhang mit dem Rechtspflege-Entlastungsgesetz wurde und wird über eine neue Einteilung der richterlichen Arbeitspensen nachgedacht: Insoweit sind Zahlen im Gespräch, wonach das Pensum eines Betreuungsrichters bei 500 neuen Fällen pro Jahr und einem Bestand von 5000 Fällen liegen soll! Man möge einmal die Phantasie spielen lassen und sich die Durchführung eines oben geschilderten Betreuungsverfahrens vor dem Hintergrund dieser Zahlen vorstellen - es dürfte auf den ersten Blick festzustellen sein, daß die Grenzen der gesetzeskonformen Anwendung des Betreuungsrechts rasch erreicht sind und spätestens das Anrollen der ersten Überprüfungswelle ${ }^{11}$ der Altfälle zum Systemzusammenbruch führen müßte - es sei denn, die Gerichte gingen zu pragmatischen, aber nicht gesetzeskonformen Anwendungen des Betreuungsrechts über ${ }^{12}$.

I I Die nach $\$ 2 \mathrm{BtG}$ funf Jahre nach Inkrafttreten dieses Gesetzes ansteht.

12 Ein modus vivendi, der - nach nicht reprasentativen, aber ubereinstimmenden Beobachtungen mehrerer mit der Materie Befaßter - bereıts jetzt verstarkt um sich greift. 